Article

\title{
Simultaneous and sensitive detection of dopamine and uric acid using a poly(L-methionine)/gold nanoparticle-modified glassy carbon electrode
}

\author{
Reza Ojani*, Jahan-Bakhsh Raoof, Ali Asghar Maleki, Saeid Safshekan \\ Electroanalytical Chemistry Research Laboratory, Faculty of Chemistry, University of Mazandaran, Babolsar, Iran
}

\section{A R T I C L E I N F O}

Article history:

Received 20 June 2013

Accepted 23 December 2013

Published 20 March 2014

\section{Keywords:}

Voltammetry

Gold nanoparticle

Poly(L-methionine)

Dopamine

Uric acid

\begin{abstract}
A B S T R A C T
A novel electrochemical sensor was fabricated by electrodeposition of gold nanoparticles on a poly(L-methionine) (PMT)-modified glassy carbon electrode (GCE) to form a nano-Au/PMT composite-modified GCE (nano-Au/PMT/GCE). Scanning electron microscopy and electrochemical techniques were used to characterize the composite electrode. The modified electrode exhibited considerable electrocatalytic activity towards the oxidation of dopamine (DA) and uric acid (UA) in phosphate buffer solution $(\mathrm{pH}=7.00)$. Differential pulse voltammetry revealed that the electrocatalytic oxidation currents of DA and UA were linearly related to concentration over the range of $5.0 \times$ $10^{-8}$ to $10^{-6} \mathrm{~mol} / \mathrm{L}$ for DA and $7.0 \times 10^{-8}$ to $10^{-6} \mathrm{~mol} / \mathrm{L}$ for UA. The detection limits were $3.7 \times 10^{-8}$ $\mathrm{mol} / \mathrm{L}$ for DA and $4.5 \times 10^{-8} \mathrm{~mol} / \mathrm{L}$ for UA at a signal-to-noise ratio of 3 . According to our experimental results, nano-Au/PMT/GCE can be used as a sensitive and selective sensor for simultaneous determination of DA and UA.
\end{abstract}

(C) 2014, Dalian Institute of Chemical Physics, Chinese Academy of Sciences. Published by Elsevier B.V. All rights reserved.

\section{Introduction}

Dopamine (DA), one of the three naturally occurring catecholamines, plays an important role as a neurotransmitter in the renal, hormonal, cardiovascular, and central nervous systems. Low levels of DA may cause brain disorders such as schizophrenia and Parkinson's disease [1-3]. Uric acid (2,6,8-trihydroxypurine, UA) is the main final product of purine metabolism in the human body. It has been shown that extreme abnormalities of UA levels are symptoms of several diseases including gout, hyperuricemia, and Lesch-Nyhan disease [4-6]. Therefore, a simple, sensitive, and accurate analytical method to quantify both DA and UA would be useful for physiological investigation as well as early diagnosis of disease [7]. Voltammetric techniques are powerful analytical tools for simultaneous determination of DA and UA in buffered solutions [8-10].
However, oxidation of DA and UA occurs at nearly the same potential, which results in an overlapping voltammetric response. It is thus impossible to discriminate between DA and UA by electrochemical methods at a bare electrode [11-13]. It is well known that chemically modified electrodes can offer better selectivity, sensitivity, time efficiency, and stability than bare ones. Therefore, many materials such as polymers [14-17], DNA-doped polymers [18], dye-doped sol-gels [19], metal oxides [20], carbon nanotubes (CNTs) [21-23], and metal nanoparticles (MNPs) [24-26] have been used to fabricate chemically modified electrodes. Among them, MNP-modified electrodes have received particular attention because of their high surface area, effective mass transport, high electrocatalytic activity, and control over local microenvironment [27,28]. However, MNPs at the electrode surface can be fragile in the absence of a stabilizing conductive material. To solve this

\footnotetext{
* Corresponding author. Tel: +98-112-5342301; Fax: +98-112-5342302; E-mail: fer-o@umz.ac.ir 
problem, the electrode surface should first be modified with a conductive stabilizing material such as ligands, CNTs, or polymers $[29,30]$. Then, the MNPs are attached to the modified electrode surface. The porous structure of conducting polymers allows the MNPs to disperse into the polymer matrix and generates additional active sites [31-33].

Gold nanoparticles (nano-Au) can bind strongly to the surface of some polymers through covalent bonding to functional groups such as $\mathrm{CN}, \mathrm{NH}_{2}$, and $\mathrm{SH}$ [34-36]. Polymers including 3-methylthiophene, thiophene, and methionine possess $\mathrm{S}$ atoms in their structure, which can readily form strong chemical bonds with nano-Au $[37,38]$. Therefore, it is expected that the surface of conducting polymer films containing $\mathrm{S}$ atoms will interact strongly with nano-Au to form good contacts via chemical bonding.

In the present study, a novel modified electrode is prepared by electrochemical deposition of nano-Au on the surface of a poly(L-methionine) (PMT)-modified glassy carbon electrode (GCE). Scanning electron microscopy (SEM) and electrochemical impedance spectroscopy (EIS) measurements indicate that nano-Au are uniformly bound onto the PMT film. The resulting nano-Au/PMT/GCE is used as a novel sensor for the simultaneous determination of DA and UA at nanomolar levels.

\section{Experimental}

\subsection{Reagents and materials}

L-Methionine, DA, and UA were purchased from Fluka and used without further purification. $\mathrm{HAuCl}_{4} \cdot 4 \mathrm{H}_{2} \mathrm{O}$ was purchased from Merck (Germany). Other reagents were of analytical grade and used without further purification. Aqueous solutions were prepared in double-distilled water, and experiments were carried out at room temperature.

\subsection{Apparatus}

Voltammetric measurements were performed using an Autolab PGSTAT 30 electrochemical analysis system equipped with the GPES 4.9 and FRA software package (Eco Chemic, the Netherlands). An electrochemical three-electrode system was used with a bare or modified GCE (3 $\mathrm{mm}$ in diameter) as the working electrode, a Pt wire as auxiliary electrode and saturated calomel electrode (SCE) as reference electrode. All pH measurements were performed by a 780 Metrohm $\mathrm{pH}$ meter.

\subsection{Preparation of a PMT film-modified GCE}

Prior to surface modification, a bare GCE was polished successively with different grades of $\mathrm{Al}_{2} \mathrm{O}_{3}$ slurry (0.05-3 micron) on synthetic cloth to obtain a mirror-like surface. The GCE was then thoroughly rinsed with pure water and sonicated in a mixture of double-distilled water and ethanol (1:1) for $5 \mathrm{~min}$. Polymerization of L-methionine on the GCE was performed using cyclic voltammetry in an aqueous solution of L-methionine $\left(2.5 \times 10^{-3} \mathrm{~mol} / \mathrm{L}\right)$ and phosphate buffer solution $(0.1 \mathrm{~mol} / \mathrm{L})$. For this purpose, six successive cycles were applied in a potential range of -0.6 to $2 \mathrm{~V}$ vs SCE at a scan rate of $100 \mathrm{mV} / \mathrm{s}$ [39]. In the first potential scan, an irreversible oxidation peak related to monomer oxidation was observed at a potential of $1.75 \mathrm{~V}$ vs SCE (data not shown). As the number of scans increased from the second cycle to the sixth, a PMT film was formed with a peak at a potential of $1.4 \mathrm{~V}$ vs SCE. Moreover, the oxidation peak current of PMT increased with the number of scans. The polymeric electrode was then rinsed thoroughly with double-distilled water. The obtained modified electrode is denoted PMT/GCE.

\subsection{Preparation of a nano-Au-modified PMT film GCE}

Nano-Au was electrochemically deposited on the PMT/GCE surface by applying a cathodic potential of $-0.23 \mathrm{~V}$ for $300 \mathrm{~s}$ in $\mathrm{HAuCl}_{4}$ solution $(0.5 \mathrm{mmol} / \mathrm{L})$ [40]. The obtained nano-Aumodified PMT film electrode was washed with double-distilled water and is denoted nano-Au/PMT/GCE.

\section{Results and discussion}

\subsection{Characterization of the nano-Au/PMT composite}

SEM images of the GCE, electropolymerized PMT film, and nano-Au/PMT composite film are shown in Fig. 1. The morphology of the bare GCE is uniform and smooth without any cracks or pits in its surface. In contrast, after electropolymeri-
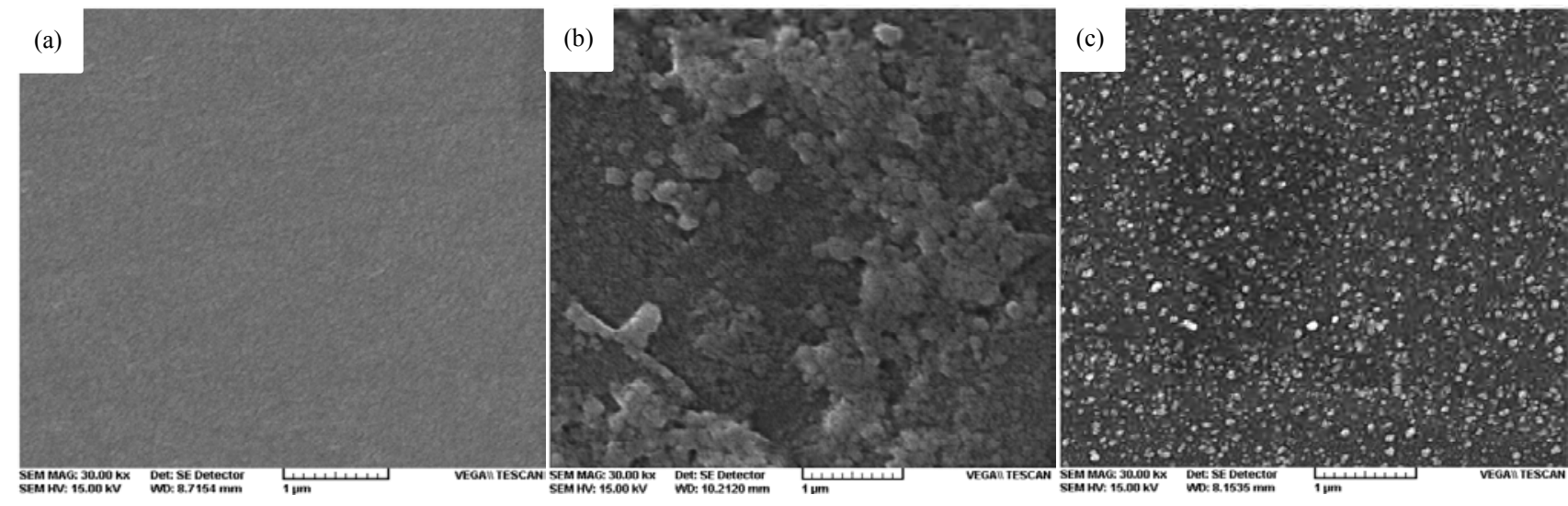

Fig. 1. SEM images of bare GCE (a), PMT/GCE (b), and nano-Au/PMT/GCE surfaces (c). 
zation, the GCE is covered by a thin and compact granular PMT film. Following deposition of nano-Au, uniform structures of nano-Au are homogeneously distributed on the surface of PMT/GCE. Nano-Au with an average size of $80 \mathrm{~nm}$ are separated from each other, so they are almost fully exposed. It should be noted that chemical bonding is the predominant process during the deposition of nano-Au onto the PMT film although physical adsorption cannot be completely ruled out [41].

EIS is an efficient tool for studying the interface properties of surface-modified electrodes [42]. The electron transfer resistance $\left(R_{\mathrm{ct}}\right)$ at the electrode surface is equal to the diameter of the semicircle obtained in EIS and can be used to describe the interface properties of the electrode. Figure 2 depicts the Nyquist diagrams of the bare GCE, PMT/GCE, and nano-Au/ PMT/GCE measured in the presence of $10 \mathrm{mmol} / \mathrm{L}$ $\mathrm{K}_{3}\left[\mathrm{Fe}(\mathrm{CN})_{6}\right] / \mathrm{K}_{4}\left[\mathrm{Fe}(\mathrm{CN})_{6}\right](1: 1)+0.1 \mathrm{~mol} / \mathrm{L} \mathrm{KCl}$ at the formal potential of $\mathrm{Fe}(\mathrm{CN})^{4-/ 3-}$. A large semicircle diameter $\left(R_{\mathrm{ct}}=6.7\right.$ $\mathrm{k} \Omega$ ) with an almost straight tail line is observed for the bare GCE. The large semicircle demonstrates the high electron transfer resistance of the GCE to the redox probe dissolved in the electrolyte solution. In the case of PMT/GCE, it can be seen that a much smaller semicircle diameter $\left(R_{\mathrm{ct}}=3.7 \mathrm{k} \Omega\right)$ appeared. This suggests that the resistance to the redox reaction was lower at PMT/GCE than that at the bare GCE. Interestingly, $R_{\text {ct }}$ for the nano-Au/PMT/GCE showed a marked decrease to about $1.9 \mathrm{k} \Omega$, which should be because the composite film of nano-Au/PMT/GCE has good conductivity. From the decrease of $R_{\mathrm{ct}}$, it can also be concluded that the nano-Au particles are distributed within the PMT film as tiny conduction centers and the resulting nano-Au/PMT composite can accelerate electron transfer between the objective molecules and electrode surface.

\subsection{Electrocatalytic oxidation of DA and UA on nano-Au/PMT/GCE}

To achieve optimal conditions for voltammetric determination of DA and UA, the main parameters related to the film formation and solution characteristics were evaluated. These parameters were the L-methionine concentration, electrodeposi-

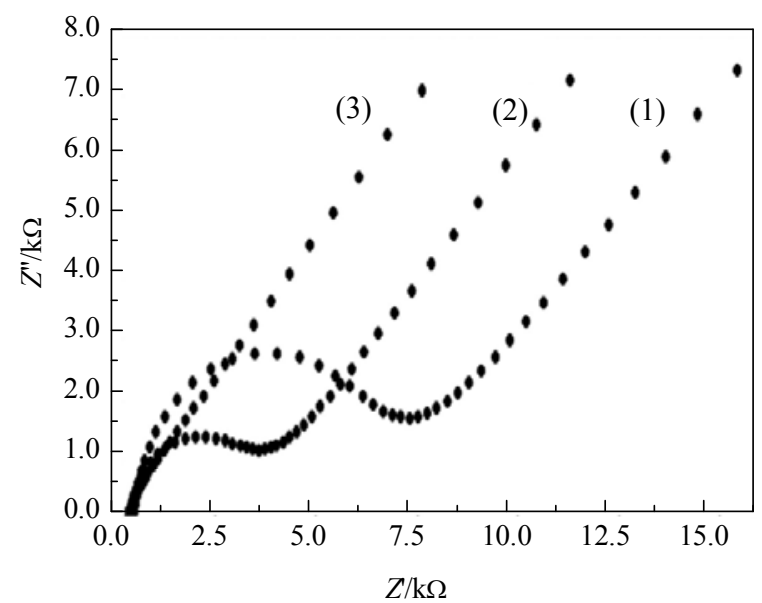

Fig. 2. Nyquist plots showing the Faradaic impedance measurements of bare GCE (1), PMT/GCE (2), and nano-Au/PMT/GCE (3) in $1 \mathrm{mmol} / \mathrm{L}$ $\mathrm{K}_{3} \mathrm{Fe}(\mathrm{CN})_{6} / \mathrm{K}_{4} \mathrm{Fe}(\mathrm{CN})_{4}(1: 1)$ containing $0.1 \mathrm{~mol} / \mathrm{L} \mathrm{KCl}$.

tion time of nano-Au and $\mathrm{pH}$ of the supporting electrolyte solution. Optimal conditions were elucidated by measuring the peak currents of both compounds with one variable at a time. We determined that the optimum conditions were as follows: $2.5 \times 10^{-3} \mathrm{~mol} / \mathrm{L}$ L-methionine, electrodeposition of nano-Au for $300 \mathrm{~s}$ and an electrolyte medium with a $\mathrm{pH}$ of 7.

Figure 3(a) shows cyclic voltammograms (CVs) of the bare GCE, PMT/GCE, and nano-Au/PMT GCE in phosphate buffer solution (PBS, $\mathrm{pH}=7.00$ ) containing $1 \times 10^{-5} \mathrm{~mol} / \mathrm{L} \mathrm{DA}$. At the bare GCE, DA exhibited very poor current response, indicating a slow electron transfer kinetics. Oxidation and subsequent reduction peaks at PMT/GCE appeared at 0.21 and $0.17 \mathrm{~V}$, respectively, indicating a quasi-reversible electrode process probably caused by the diffusion barrier of the polymeric film. Similar peak potentials were obtained with the nano-Au/PMT/ GCE electrode with higher peak currents. It seems tht the electrochemical reaction kinetics was improved by the nano-Au inserted into the polymeric film matrix. As a result, nano-Au/ PMT/GCE exhibited a small peak-to-peak separation $\left(\Delta E_{\mathrm{p}}=\right.$ 0.04) with almost reversible electrochemical behavior. The
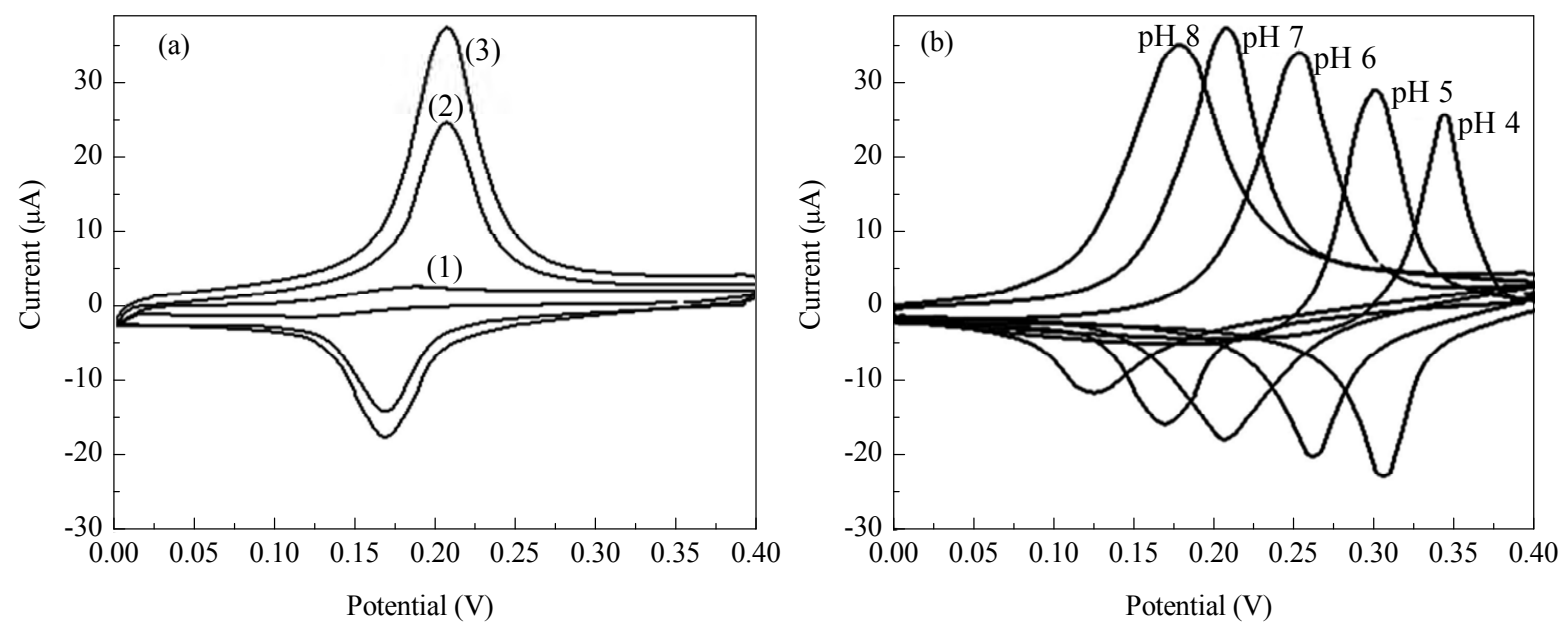

Fig. 3. (a) CVs obtained at bare GCE (1), PMT/GCE (2), and nano-Au/PMT/GCE (3) in the presence of $1 \times 10^{-5} \mathrm{~mol} / \mathrm{L} \mathrm{DA}$ with a scan rate of 50 mV/s in $0.1 \mathrm{~mol} / \mathrm{L} \mathrm{PBS}$ at $\mathrm{pH}=7.00$. (b) CVs of nano-Au/PMT/GCE in $0.1 \mathrm{~mol} / \mathrm{L} \mathrm{PBS}$ containing $1 \times 10^{-5} \mathrm{~mol} / \mathrm{L} \mathrm{DA} \mathrm{at} \mathrm{different} \mathrm{pH.}$ 
peak current was also five times higher than that of the bare GCE and twice that of PMT/GCE. The peak characteristics clearly indicated electrocatalytic oxidation of DA at the modified electrodes because of its more active surface than that of the bare GCE. This marked enhancement of peak current at the surface of the modified electrodes confirms that both PMT and nano-Au facilitate the electrochemical reactions. The influence of solution $\mathrm{pH}$ on the electrochemistry of DA oxidation was also studied (Fig. 3(b)). The anodic peak potential shifted linearly with increasing solution $\mathrm{pH}$ with a slope of about $0.052 \mathrm{~V} / \mathrm{pH}$. The magnitude of the slope for DA oxidation suggests that two protons were involved in the electrode reaction.

CVs of UA in PBS at the bare GCE, PMT/GCE, and nano-Au/ PMT/GCE are presented in Fig. 4(a). UA shows quasi-reversible electrochemical behavior with an anodic peak potential of about $0.37 \mathrm{~V}$. All three voltammograms display a small cathodic peak at $0.34 \mathrm{~V}$, indicating slow cathodic electron transfer. Under identical conditions, both PMT/GCE and nano-Au/PMT/ GCE show a marked increase of peak current to UA oxidation compared with that of the bare GCE. This suggests more efficient oxidation of UA at nano-Au/PMT/GCE than at the bare
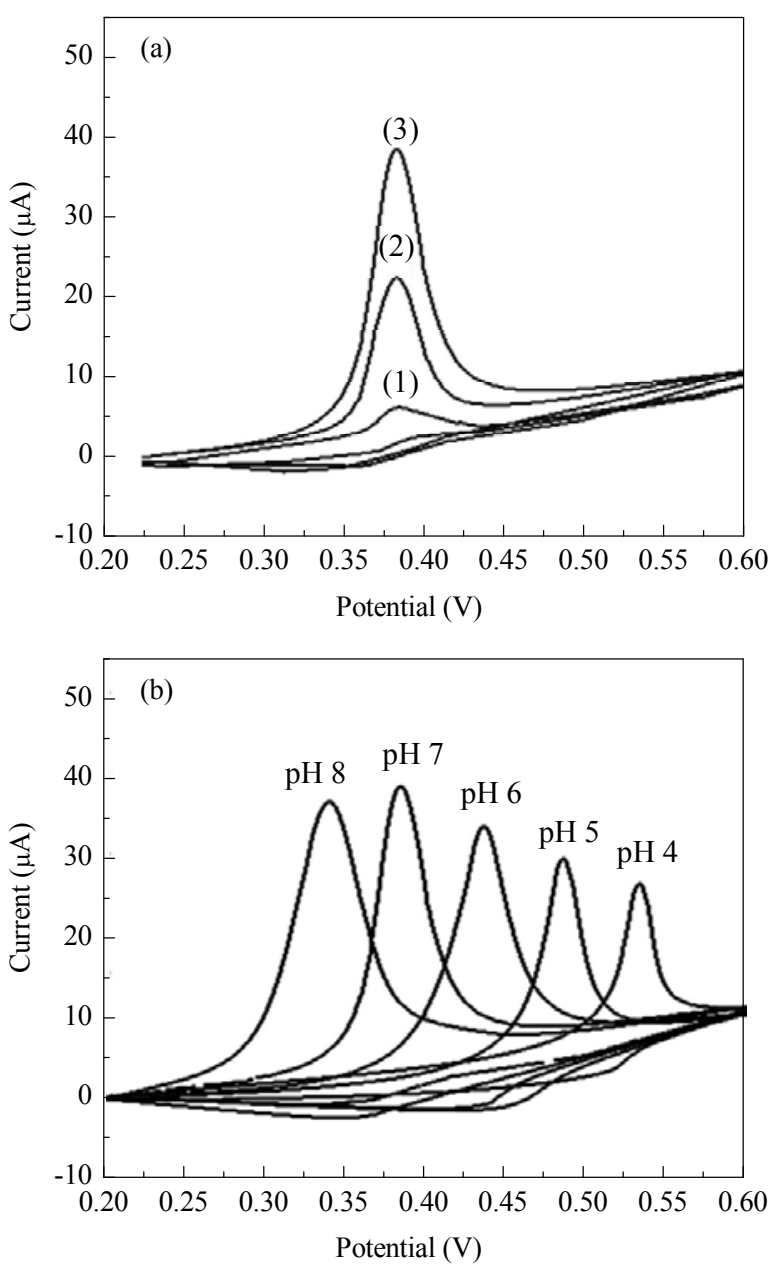

Fig. 4. (a) CVs obtained at bare GCE (1), PMT/GCE (2), and nano-Au/PMT/GCE (3) in the presence of $1 \times 10^{-5} \mathrm{~mol} / \mathrm{L}$ UA with a scan rate of $50 \mathrm{mV} / \mathrm{s}$ in $0.1 \mathrm{~mol} / \mathrm{L} \mathrm{PBS}$ at $\mathrm{pH}=7.00$. (b) CVs of nano-Au/PMT/GCE in $0.1 \mathrm{~mol} / \mathrm{L}$ PBS containing $1 \times 10^{-5} \mathrm{~mol} / \mathrm{L} \mathrm{UA}$ at different $\mathrm{pH}$.
GCE, probably because of the larger effective surface area supplied by both the polymeric film and nano-Au on the GCE. Solution $\mathrm{pH}$ has a pronounced effect on the electrochemical response of UA (Fig. 4(b)). The oxidation peak of UA shifted to more negative potential as $\mathrm{pH}$ increased. The slope of about $0.051 \mathrm{~V} / \mathrm{pH}$ for UA oxidation showed that two protons were involved in the electrode reaction at nano-Au/PMT/GCE. The highest oxidation current was obtained at $\mathrm{pH}=7.00$; the oxidation peak current was decreased at both lower and higher $\mathrm{pH}$ because of the instability of UA under both acidic and alkaline solutions.

\subsection{Separation of the electrochemical responses of DA and UA at modified electrodes}

Figure 5 shows CVs measured for a mixture of $1 \times 10^{-5}$ mol/L DA and $1 \times 10^{-5} \mathrm{~mol} / \mathrm{L} \mathrm{UA}$ at the bare GCE, PMT/GCE, and nano-Au/ PMT/GCE. Two separate broad peaks were obtained in forward scans for PMT/GCE and nano-Au/PMT/GCE. In contrast, convolution of the oxidation peaks limited the selectivity and sensitivity of simultaneous determination of DA and UA at the bare GCE. Modification of the GCE surface with a PMT film and deposition of nano-Au resolved the merged voltammetric peaks into two well-defined oxidation peaks at 0.23 and $0.37 \mathrm{~V}$ for DA and UA, respectively. The separation of the two oxidation peaks allows the simultaneous determination of DA and UA in a mixed solution. Deposition of nano-Au also enhanced the catalytic activity of PMT/GCE for oxidation of a mixture of DA and UA in terms of both oxidation potential and peak current. In fact, the presence of nano-Au improved both the mass and electronic transportation rates of the reaction, which lowers overpotential and enhances the oxidation currents of DA and UA.

\subsection{Differential pulse voltammetric determination of UA and $D A$}

Differential pulse voltammetry (DPV) can discriminate against background noise and offer much higher current sensi-

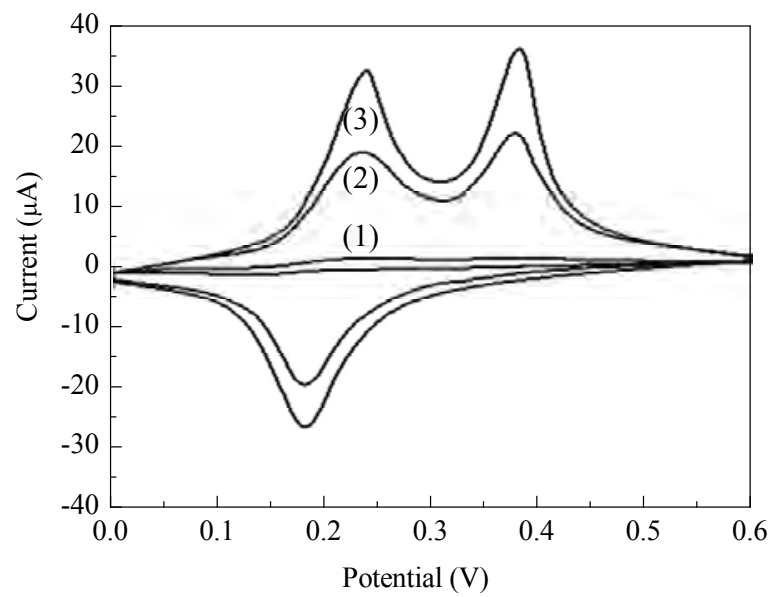

Fig. 5. CVs of $1 \times 10^{-5} \mathrm{~mol} / \mathrm{L} \mathrm{DA}+1 \times 10^{-5} \mathrm{~mol} / \mathrm{L} \mathrm{UA}$ at bare GCE (1), PMT/GCE (2), and nano-Au/PMT/GCE (3) at a scan rate of $50 \mathrm{mV} / \mathrm{s}$ in $0.1 \mathrm{~mol} / \mathrm{L} \mathrm{PBS}$ at $\mathrm{pH}=7.00$. 
tivity and better peak separation than cyclic voltammetry. DPV and calibration curves for various concentrations of DA and UA in PBS at $\mathrm{pH}=7.00$ are displayed in Figs. 6 and 7, respectively. A linear response was obtained between the peak current and concentration of DA (Fig. 6(b)) in the range of $5.0 \times 10^{-8}$ to $1 \times$ $10^{-6} \mathrm{~mol} / \mathrm{L}$ with a detection limit of $3.7 \times 10^{-8}(\mathrm{~s} / \mathrm{n}=3)$. With regard to UA (Fig. 7(b)), the linear range was from $7.0 \times 10^{-8}$ to $1 \times 10^{-6} \mathrm{~mol} / \mathrm{L}$ with an equation of $i_{\mathrm{pa}}(\mu \mathrm{A})=19.55+1.332$ $(\mu \mathrm{mol} / \mathrm{L})\left(R^{2}=0.999\right)$. The detection limit for UA was $4.5 \times 10^{-8}$ $\mathrm{mol} / \mathrm{L}(s / n=3)$.

\subsection{Selective determination of DA in the presence of a fixed concentration of UA}

Figure 6 displays DPVs that were obtained for different concentrations of DA in the presence of $1 \times 10^{-6} \mathrm{~mol} / \mathrm{L} \mathrm{UA}$ at nano-Au/PMT/GCE. Oxidation peaks with adequate resolution for both compounds were obtained at $0.2 \mathrm{~V}$ for DA and $0.36 \mathrm{~V}$ for UA using nano-Au/PMT/GCE. The oxidation peak current of DA increased linearity $\left(R^{2}=0.999\right)$ with increasing concentration in the range of $5.0 \times 10^{-8}$ to $1 \times 10^{-6} \mathrm{~mol} / \mathrm{L}$, whereas the
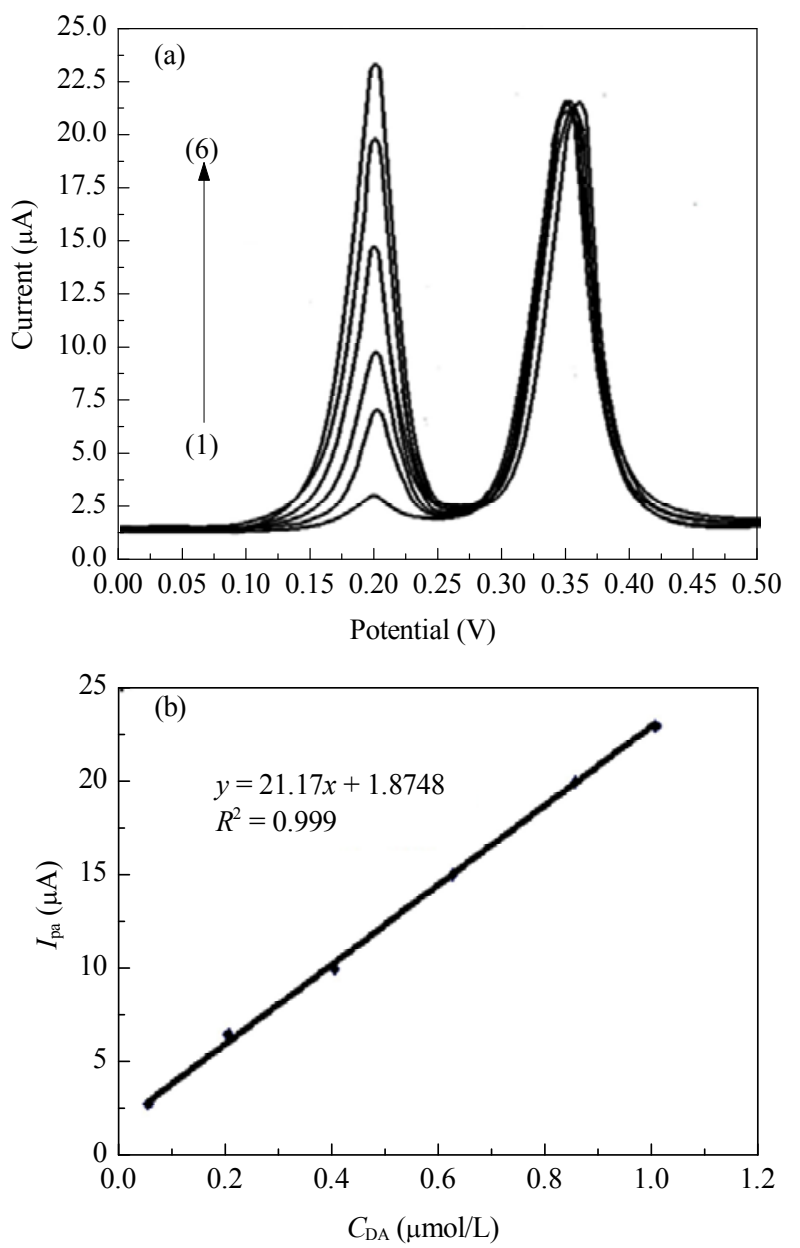

Fig. 6. (a) DPVs of DA in the presence of UA $\left(1 \times 10^{-6} \mathrm{~mol} / \mathrm{L}\right)$ at nano-Au/PMT $/ \mathrm{GCE}$ in PBS $(0.1 \mathrm{~mol} / \mathrm{L})$ at $\mathrm{pH}=7.00$ with a scan rate of $20 \mathrm{mV} / \mathrm{s}$ and pulse amplitude of $50 \mathrm{mV}$. Concentration of DA (mol/L): (1) $5.0 \times 10^{-8}$; (2) $2.0 \times 10^{-7}$; (3) $4.0 \times 10^{-7}$; (4) $6.2 \times 10^{-7}$; (5) $8.5 \times 10^{-7}$; (6) $1.0 \times 10^{-6}$. (b) Plot of oxidation peak current against concentration of DA.
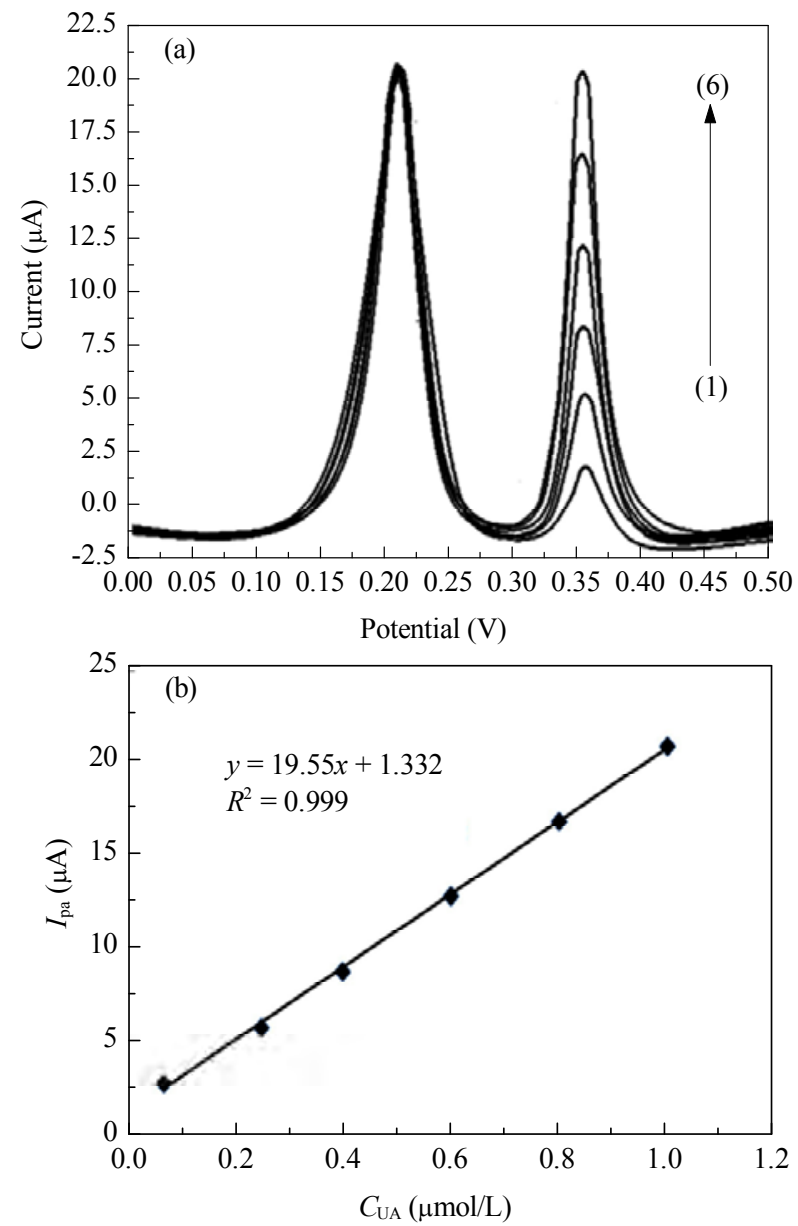

Fig. 7. (a) DPVs of UA in the presence of DA $\left(1 \times 10^{-6} \mathrm{~mol} / \mathrm{L}\right)$ at nano-Au/PMT/GCE in PBS ( $0.1 \mathrm{~mol} / \mathrm{L})$ at $\mathrm{pH}=7.00$ with a scan rate of $20 \mathrm{mV} / \mathrm{s}$ and pulse amplitude of $50 \mathrm{mV}$. Concentration of UA (mol/L): (1) $7.0 \times 10^{-8}$; (2) $2.5 \times 10^{-7}$; (3) $4.0 \times 10^{-7}$; (4) $6.0 \times 10^{-7}$; (5) $8.0 \times 10^{-7}$; (6) $1 \times 10^{-6}$. (b) Plot of oxidation peak current against concentration of UA.

voltammetric peak current of UA remained the same. Similarly, voltammetric determination of UA was carried out in the presence of DA at a fixed concentration of $1 \times 10^{-6} \mathrm{~mol} / \mathrm{L}$. As shown in Fig. 7, an increase in the concentration of UA did not cause an obvious change in the oxidation current of DA. The peak current of UA increased linearly with UA concentration $\left(7.0 \times 10^{-8}\right.$ to $1 \times 10^{-6} \mathrm{~mol} / \mathrm{L}$ ).

Table 1 summarizes the analytical performance of nano-Au/ PMT/GCE and some previously reported modified electrodes in terms of linearity and detection limit. Improved or comparable performance for the simultaneous determination of UA and DA can be achieved using the nano-Au/PMT/GCE compared with other modified electrodes.

\subsection{Reproducibility, repeatability and stability}

We checked the reproducibility and stability of nano-Au/ PMT/GCE over time. The performance of nano-Au/PMT/GCE for the detection of DA was tested in PBS ( $\mathrm{pH}=7.00)$ for a long period. Over the first 2 days, the signal showed a $2 \%$ decrease 
Table 1

Comparison of the analytical performance of several modified electrodes for the simultaneous determination of DA and UA.

\begin{tabular}{|c|c|c|c|c|c|c|}
\hline \multirow{2}{*}{ Modified electrode } & \multirow{2}{*}{ Method } & \multicolumn{2}{|c|}{ Linear range $(\mu \mathrm{mol} / \mathrm{L})$} & \multicolumn{2}{|c|}{ Detection limit $(\mu \mathrm{mol} / \mathrm{L})$} & \multirow{2}{*}{ Ref. } \\
\hline & & DA & UA & DA & UA & \\
\hline$\overline{\mathrm{Pd}_{3} \mathrm{Pt}_{1} / \mathrm{PDDA}-\mathrm{RGO} / \mathrm{GCE}}$ & DPV & $4-200$ & $4-400$ & 0.04 & 0.10 & [43] \\
\hline CNT/PEDOT/GCE & DPV & $10-330$ & $10-250$ & 10 & 10 & [44] \\
\hline AuNPs- $\beta$-CD-Gra/GCE & SWP & $0.50-150$ & $0.5-60$ & 0.15 & 0.21 & {$[45]$} \\
\hline PDDA@HCNT/GCE & DPV & $0.25-10$ & $5.0-175$ & 0.08 & 0.22 & [46] \\
\hline PGE & DPV & $0.15-15$ & $0.3-150$ & 0.033 & 0.12 & [47] \\
\hline HNCMS/GCE & DPV & $3-75$ & $5-30$ & 0.04 & 0.02 & [48] \\
\hline SWCNH/GCE & LSV & $0.2-3.8$ & $0.06-10$ & 0.06 & 0.02 & [49] \\
\hline Nano-Au/PMT/GCE & DPV & $0.05-1$ & $0.07-1$ & 0.037 & 0.045 & this work \\
\hline
\end{tabular}

compared with its initial response. After 8 days, the current response decreased by about $5 \%$ and in the following 2 weeks, the decrease was $12 \%$. Nano-Au/PMT/GCE retained $88 \%$ of its original activity after 2 weeks and continued to exhibit excellent response to both UA and DA. Therefore, nano-Au/PMT/ GCE is not readily fouled by the oxidation products of UA and DA and exhibits high stability for the simultaneous determination of UA and DA. The experiments were repeated for DA and UA mixtures and similar stable signals were obtained. These results confirm that nano-Au/PMT/GCE is stable and can be used in voltammetric studies for the selective and sensitive determination of DA and UA.

\section{Conclusions}

A new modified electrode, nano-Au/PMT/GCE, was fabricated by electrodeposition of nano-Au into a PMT matrix formed on a GCE and used for the simultaneous determination of DA and UA. The electrochemical activity of the modified electrode towards DA and UA oxidation was improved by the formation of a uniform PMT film containing nano-Au on the electrode surface. DPV measurements revealed the detection limits of DA and UA on nano-Au/PMT/GCE were $3.7 \times 10^{-8}$ and $4.5 \times 10^{-8} \mathrm{~mol} / \mathrm{L}$, respectively. The modified electrode showed excellent sensitivity, selectivity, and anti-fouling properties for the voltammetric determination of DA and UA individually or simultaneously. This electrode can be used for DA and UA determination in real samples with satisfactory results.

\section{References}

[1] Smith T E, Devlin T M Ed. Textbook of Biochemistry with Clinical Correlations. New York: Wiley/Liss, 1992

[2] O'Neill R D. Analyst, 1994, 119: 767

[3] Downard A J, Roddick A D, Bond A M. Anal Chim Acta, 1995, 317: 303

[4] Miland E, Ordieres A J M, Blanco P T, Smyth M R, Fagain C O. Talanta, 1996, 43: 785

[5] Shi K, Shiu K K. Electroanalysis, 2001, 13: 1319

[6] Zheng L Z, Wu S G, Lin X Q, Nie L, Rui L. Electroanalysis, 2001, 13: 1351

[7] Paschla L A, Reynolds D L, Wright D S, Kissinger P T. J Assoc Offic Anal Chem, 1987, 70: 1

\section{Graphical Abstract}

Chin. J. Catal., 2014, 35: 423-429 doi: 10.1016/S1872-2067(14)60022-X

Simultaneous and sensitive detection of dopamine and uric acid using a poly(L-methionine)/gold nanoparticle-modified glassy carbon electrode

Reza Ojani*, Jahan-Bakhsh Raoof, Ali Asghar Maleki, Saeid Safshekan

University of Mazandaran, Iran
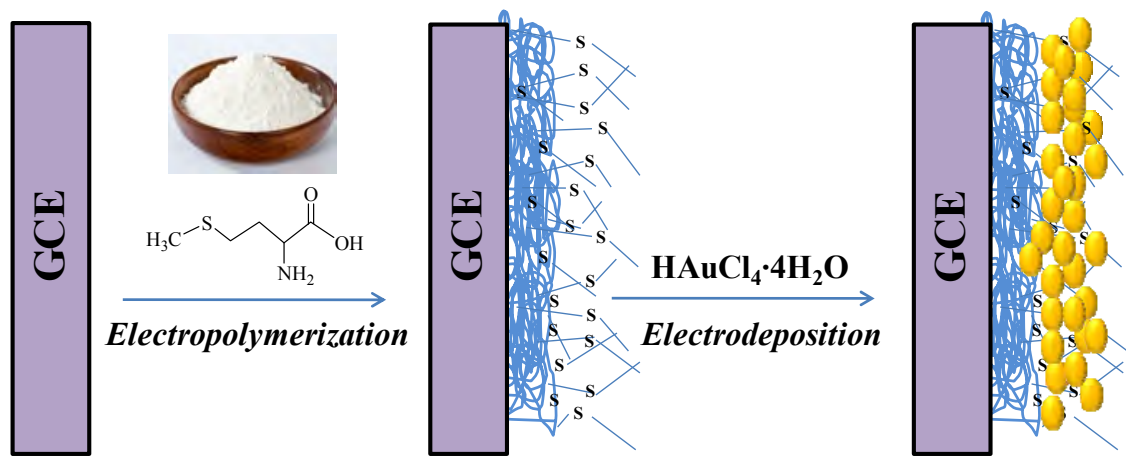

A glassy carbon electrode modified with gold nanoparticles and poly(L-methionine) exhibited high electrocatalytic activity towards the oxidation of dopamine and uric acid. This electrode allowed sensitive and selective determination of dopamine and uric acid at nanomolar levels. 
[8] Dutt J S N, Cardosi M F, Livingstone C, Davis J. Electroanalysis, 2005, 17: 1233

[9] Rivas G A, Rubianes M D, Rodrigues M C, Ferreyra N F, Lugue G L, Pedano M L, Miscoria S A, Parrado C. Talanta, 2007, 74: 291

[10] Bravo R, Hsueh C C, Jaramillo A, Brajter-Toth A. Analyst, 1998, 123: 1625

[11] Giddings J C, Yang F J, Myers M N. Anal Chem, 1976, 48: 1126

[12] Stamford J A, Justice J B Jr. Anal Chem, 1996, 68: 359A

[13] Gonon F, Buda M, Cespuglio R, Jouvet M, Pajol J F. Nature, 1980, 286: 902

[14] Jin G Y, Zhang Y Z, Cheng W X. Sens Actuators B, 2005, 107: 528

[15] Zhao H, Zhang Y, Yuan Z. Anal Chim Acta, 2001, 441: 117

[16] Li J, Lin X Q. Sens Actuators B, 2007, 124: 486

[17] Mathiyarasu J, Senthilkumar S, Phani K L N, Yegnaraman V. Mater Lett, 2008, 62: 571

[18] Lu L P, Lin X Q. Electrochem Commun, 2008, 10: 704

[19] Khoo S B, Chen F. Anal Chem, 2002, 74: 5734

[20] Shakkthivel P, Chen S M. Biosens Bioelectron, 2007, 22: 1680

[21] Ensafi A A, Bahrami H, Karimi-Maleh H, Mallakpour Sh. Chin J Catal (催化学报), 2012, 33: 1919

[22] Liu A, Honma I, Zhou H. Biosens Bioelectron, 2007, 23: 74

[23] Jiao S F, Li M G, Wang C, Chen D L, Fang B. Electrochim Acta, 2007, 52: 5939

[24] Sivanesan A, Kannan P, Abraham John S. Electrochim Acta, 2007, 52: 8118

[25] Raj C R, Okajima T, Ohsaka T.J Electroanal Chem, 2003, 543: 127

[26] Zhang L Y, Yuan R, Chai Y Q Li X L. Anal Chim Acta, 2007, 596: 99

[27] Safavi A, Maleki N, Tajabadi F, Farjami E. Electrochem Commun, 2007, 9: 1963

[28] Shipway A N, Lahav M, Willner I. Adv Mater, 2000, 12: 993
[29] Muraviev D N. Contributions to Science, 2005, 3: 19

[30] Ohnuki Y, Ohsaka T, Matsuda H, Oyama N. J Electroanal Chem Interfacial Electrochem, 1983, 158: 55

[31] Tian S J, Liu J Y, Zhu T, Knoll W. Chem Mater, 2004, 16: 4103

[32] Zhang L J, Wan M X. J Phys Chem B, 2003, 107: 6748

[33] Davies R, Schurr G A, Meenan P, Nelson R D, Bergna H E, Brevett C A S, Goldbaum R H. Adv Mater, 1998, 10: 1264

[34] Bose C S C, Rajeshwar K.J Electroanal Chem, 1992, 333: 235

[35] Li J, Lin X Q. Microchem J, 2007, 87: 41

[36] Liu Y C, Lee H T, Yang S J. Electrochim Acta, 2006, 51: 3441

[37] Roux S, Soler-Illia G J A A, Demoustier-Champagne S, Audebert P, Sanchez C. Adv Mater, 2003, 15: 217

[38] Chen W, Li C M, Chen P, Sun C Q. Electrochim Acta, 2007, 52: 2845

[39] Ma W, Sun D M. Russian J Electrochem, 2007, 43: 1382

[40] Li J, Lin X Q. Anal Chim Acta, 2007, 596: 222

[41] Cho S H, Park S M. J Phys Chem B, 2006, 110: 25656

[42] Katz E, Willner I. Electroanalysis, 2003, 15: 913

[43] Yan J, Liu S, Zhang Z Q He G W, Zhou P, Liang H Y, Tian L L, Zhou X M, Jiang H J. Colloids Surf B, 2013, 111: 392

[44] Lin K C, Tsai T H, Chen S M. Biosens Bioelectron, 2010, 26: 608

[45] Tian X Q, Cheng C M, Yuan H Y, Du J, Xiao D, Xie S P, Choi M M F. Talanta, 2012, 93: 79

[46] Zhang B Y, Huang D K, Xu X B, Alemu G, Zhang Y B, Zhan F, Shen Y, Wang M K. Electrochim Acta, 2013, 91: 261

[47] Alipour E, Majidi M R, Saadatirad A, Golabi S M, Alizadeh A M. Electrochim Acta, 2013, 91: 36

[48] Xiao C H, Chu X C, Yang Y, Li X, Zhang X H, Chen J H. Biosens Bioelectron, 2011, 26: 2934

[49] Zhu S Y, Li H J, Niu W X, Xu G B. Biosens Bioelectron, 2009, 25: 940 